\title{
Structural Stress Identification Using Fuzzy Pattern Recognition and Information Fusion Technique
}

\author{
Jun Teng, Ting Zhang and Wei Lu \\ Shenzhen Graduate School, Harbin Institute of Technology, Shenzhen, China
}

\begin{abstract}
In order to ensure the service security of space structures under wind load, the stress identification method based on the combination of fuzzy pattern recognition and information fusion technique is proposed, in which the measurements of limited strain sensors arranged on the structure are used. Firstly, the structure is divided into several regions according to the similarity and the most unfavorable region is selected to be the key region for stress identification, while the different numbers of the strain sensors are located on the key region and the normal regions; secondly, the different stress distributions of the key region are obtained based on the measurements of the strain sensors located on the key region and the normal regions separately, in which the fuzzy pattern recognition is used to identify the different stress distributions; thirdly, the stress distributions obtained by the measurements of sensors in normal regions are selected to calculate the synthesized stress distribution of the key region by D-S evidence theory; fourthly, the weighted fusion algorithm is used to assign the different fusion coefficients to the selected stress distributions obtained by the measurements of the normal regions and the key region, while the synthesized stress distribution of the key region can be obtained. Numerical study on a lattice shell model is carried out to validate the reliability of the proposed stress identification method. The simulated results indicate that the method can improve identification accuracy and be effective by different noise disturbing.
\end{abstract}

Key words: Stress identification, Fuzzy pattern recognition, information fusion technique.

\section{Introduction}

Large space structures are usually the important symbols in cities, which are wind-sensitive because of their small damping ratio and low frequency in vibration. Therefore, the safety and reliability of the large space structures under wind load is one of the most important research problems. Structural health monitoring is a method which can obtain the structural responses and give the estimation of the working status of the structure, while different types of sensors are arranged on the structure. Recently, the researchers [1-5] put emphasis on realizing objectives and functions of structural health monitoring system using limited measurements of sensors. For example, Ming Liu et al. [6] assessed the reliability of bridge through the long-term monitoring measurements of strain sensors under traffic loads and researched the

Corresponding author: Jun Teng, $\mathrm{PhD}$, professor, research field: structural health monitoring. E-mail: tengj@hit.edu.cn. security limit using the actual traffic conditions and measurements of strain sensor on Wisconsin Rive Bridge in United States.

Key region is defined as the unfavorable region with higher stress level than the normal regions of the structure, which is related to the safety of the structure directly. Generally, the stress distribution of the key region is obtained by the measurements of limited sensors located on the key region directly, in which the stresses of elements without sensors locating on can not be obtained. In addition, it is impossible to measure the stresses of all the elements in the key region due to the requirements in economic and the construction of the structural health monitoring system. Therefore, the stress identification method based on measurements of limited strain sensors is proposed.

In the structural health monitoring system of Shenzhen Citizen Center in China, the stress fields of brace steel brackets are identified by the limited 
measurements of strain sensors located on the key points [7]. However, the measurements of strain sensors located on the normal regions should also be used to obtain the stress distribution identification of the key region, which can provide much more known measurements and reduce the incompleteness of information existing in structural health monitoring system. Information fusion technique can fuse the measurements of strain sensors located on key region and the normal regions, and give synthesized information for identification, eliminate redundancy and contradictions that may exist. Information fusion technique has been used in structural damage identification, while it is demonstrated that the identification results are better than that only using one sensor information source by numerical examples $[8,9]$.

The objective of the research is to develop a stress identification method using fuzzy pattern recognition and information fusion technique, while the acquisition for the stress distribution of the key region is discussed using the measurements of strain sensors located on both the key region and the normal regions. Correspondingly speaking, the acquisition for the stress distribution of the key region using measurements of strain sensors located on the key region is called the key region identification; and the acquisition for the stress distribution of the key region using measurements of strain sensors located on the normal regions is called the normal region identification. Firstly, the key region identification and the normal region identification are obtained by the fuzzy pattern recognition; secondly, information fusion technique is used to give the synthesized stress distribution of the key region; finally, the stress distribution identification for a shell structure is simulated and the method is proofed to be effective.

\section{Basic Process}

The proposed method is divided into early-stage preparation part and real-time monitoring part, which is shown in Fig. 1. In the early-stage preparation part, firstly, the key region identification and the normal region identification are obtained using fuzzy pattern recognition; secondly, the selected normal region identification results are decided using the D-S evidence theory; thirdly, the different fusion coefficients for the key region identification and the selected normal identification are assigned using the

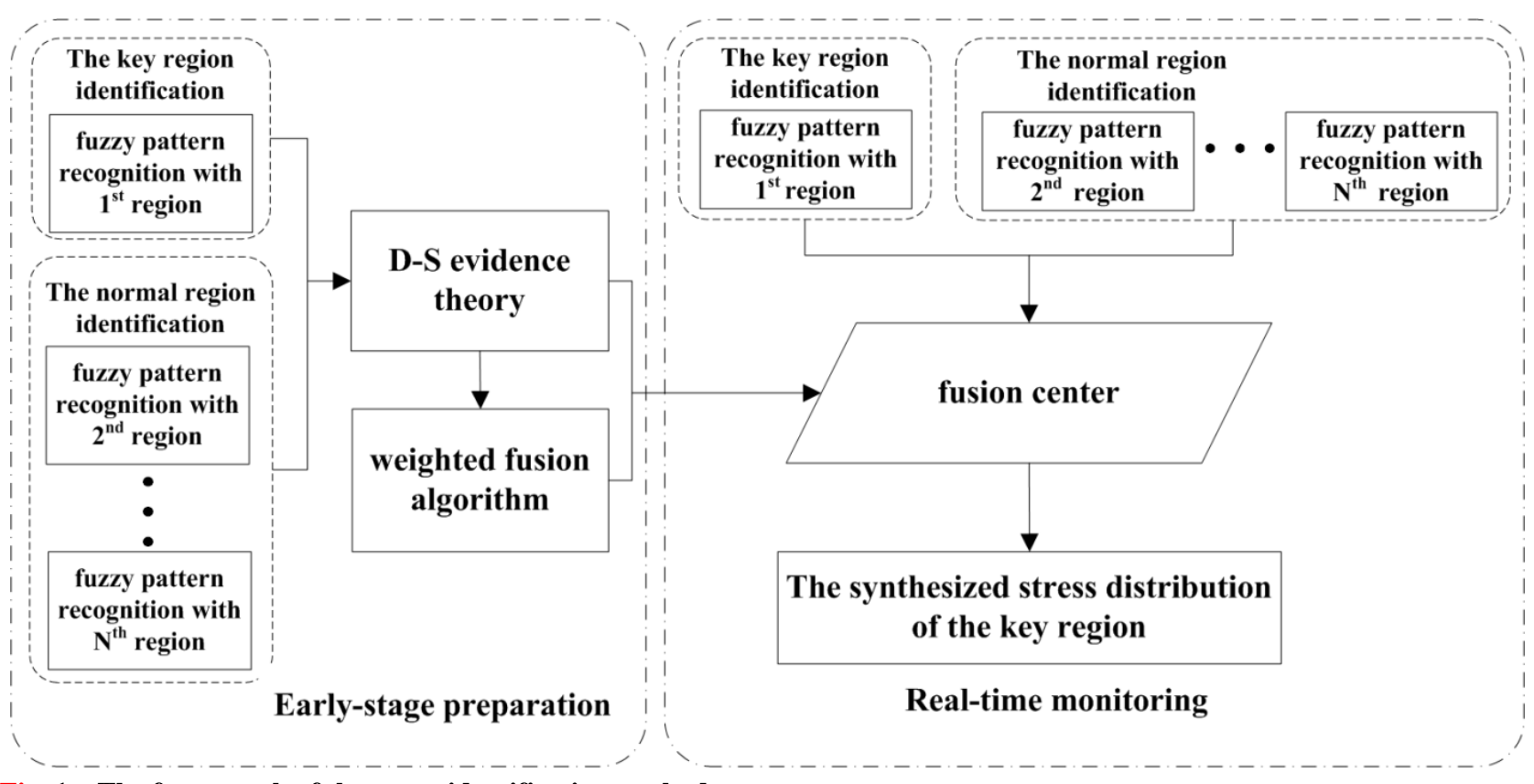

Fig. 1 The framework of the stress identification method. 
weighted fusion algorithm; finally, all the results are saved in the fusion center for obtaining the synthesized stress distribution of the key region in real-time. In the real-time monitoring part, firstly, the key region identification and the normal region identification are acquired using fuzzy pattern recognition; secondly, the synthesized stress distribution of the key region can be obtained with different fusion coefficients.

\section{Fuzzy Pattern Recognition}

The pattern recognition aims to classify the new pattern into some pattern in the standard pattern library based on a priori knowledge, in which the feature vector is extracted to represent the characteristics of the pattern and the fuzzy neartude is used to measure the similarity between new pattern and some pattern in the standard pattern library. To represent the global characteristics of the feature vectors, the fuzzy set constructed by the membership function of each element in feature vector is used to calculate the fuzzy neartude between patterns.

In the paper, the feature vectors of pattern recognition can be obtained by the measurements of strain sensors located on the key region and the normal regions. The standard pattern library is formed by calculating the different stress distributions of the key regions under different load cases in the finite element analysis, while the domain $U$ of fuzzy pattern recognition is formed by the feature vectors under different load cases. In order to reflect the characteristics of the stress distributions of the key region better, the variation of stresses in measured elements should be sensitive to the change of load cases, so the elements with larger means and variation coefficients in stress distributions are selected to located sensors on.

\subsection{Membership Function}

The $i^{\text {th }}$ pattern in the standard pattern library, the feature vector can be denoted as:

$$
\sigma^{i}=\left[\sigma_{1}^{i}, \sigma_{2}^{i}, \cdots, \sigma_{n}^{i}\right]
$$

The membership function of the stress in the $j^{\text {th }}$ measured element in the feature vector can be expressed as [7]:

$$
\mu_{j}^{i}=\frac{\sigma_{j}^{i}-\sigma_{j \min }^{i}}{\sigma_{j \max }^{i}-\sigma_{j \min }^{i}} \quad j=1,2, \cdots, n
$$

where, $\sigma_{j}$ represents the stress of the $j^{\text {th }}$ measured element of the $i^{\text {th }}$ pattern, $\sigma_{j \max }$ and $\sigma_{j \min }$ are the maximum and minimum stresses of the $j^{\text {th }}$ measured element in the domain $U$. In this way, corresponding with domain $U$, the fuzzy subset $A_{i}$ of the $i^{\text {th }}$ patter is:

$$
A_{i}=\left[\mu_{1}, \mu_{2}, \cdots, \mu_{n}\right]
$$

The standard pattern library is composed of $\mathrm{m}$ patterns, whose fuzzy set is denoted as $A$,

$$
A=\left\{A_{1}, A_{2}, \cdots, A_{m}\right\}
$$

And the fuzzy set $B$ to be recognized can also be calculated in the same way. If $B$ is close to $A_{i}$, then $B$ is classified into $A_{i}$.

\subsection{The Principle of Classification}

The fuzzy neartude which can measure the nearness of two fuzzy subsets is denoted as [10]:

$$
D\left(A_{i}, B\right)=\frac{2 \sum_{j=1}^{n} \min \left(\mu_{A_{i}}\left(x_{j}\right), \mu_{B}\left(x_{j}\right)\right)}{\sum_{j=1}^{n} \mu_{A_{i}}\left(x_{j}\right)+\sum_{j=1}^{n} \mu_{B}\left(x_{j}\right)}
$$

where, $B\left(\mu_{j}\right)$ represents the membership function of the stress in the $j^{\text {th }}$ measured elements in the pattern to be recognized. The fuzzy neartudes of fuzzy subsets between the pattern to be recognized and the patterns in the standard pattern library can be acquired by Eq. (5).

In the original fuzzy pattern recognition method, the pattern of stress distribution to be recognized is classified into some pattern with the largest fuzzy neartude in the standard pattern library, that is to say, the stress distribution of the key region is the stress distribution of the pattern with the largest fuzzy neartude. However, the differences among the first few patterns with larger fuzzy neartudes are very small, that is to say, such patterns in the library can 
also represent a certain feature of the pattern to be recognized. Therefore, in the paper, the first few patterns with larger fuzzy neartudes are considered to decide the weighting coefficients for classifying the new pattern, while an improved method is proposed and the corresponding identified stress is known as:

$$
\sigma_{i}^{\prime}=\frac{\sum_{j=1}^{m} D_{j} \sigma_{i j}}{\sum_{j=1}^{m} D_{j}}
$$

where, $\sigma_{i}{ }^{\prime}$ is the stress identification result of the $i^{\text {th }}$ measured element in the key region, $D_{j}$ is the $j^{\text {th }}$ largest fuzzy neartude, $\sigma_{i j}$ is the stress of the $i^{\text {th }}$ measured element in the pattern with $j^{\text {th }}$ largest fuzzy neartude, $m$ is the number of patterns which participate in the weighted average.

\section{Information Fusion}

\subsection{D-S Evidence Theory}

D-S evidence theory [11], a probability-based information fusion classification algorithm, is useful when the information sources contributing information cannot associate a 100 percent probability of certainty to their output decisions. The D-S evidence theory is used to select the normal region identification results which are applied to calculate the synthesized stress distribution of the key region.

The hypothesis space is made of a finite set of $n$ mutually exclusive and exhaustive propositions, which is called the frame of discernment and denoted by $\Theta$. A power set of $2^{\Theta}$ is the set of all the subsets of $\Theta$ including itself and a null set $\Phi$. Each subset is called a focal element. The basic probability assignment (BPA) is critical variable of evidence theory. The BPA of any subset $A$ is represented as $m(A)$, which defines a mapping of $2^{\Theta}$ to the interval between 0 and 1 . The value 0 and 1 indicate no belief and total belief in a proposition separately, and any value between these two limits indicates different partial beliefs. Formally, the description of BPA can be represented as:

$$
\begin{aligned}
& m: 2^{\Theta} \rightarrow[0,1] \\
& m(\phi)=0 \\
& \sum_{A \subseteq 2^{\Theta}} m(A)=1
\end{aligned}
$$

The assigned probability to $\Theta$ is considered as the amount of uncertainty within the BPA.

In the paper, the BPA is acquired by statistical evidence method [12]. If a group of evidence is acquired on the results of statistical test, then it is called statistical evidence. Statistical evidence is the usage of D-S evidence theory in statistical problems, which is a new method Shafer used to handle statistical problem, and is an attempt using non-statistical methods to solve statistical problem.

The observation of statistical testing is determined by a group of probability models $\left\{p_{o} \mid o \in \Theta\right\}$, in which $\Theta$ is the frame of discernment, $p_{o}$ is the probability when the proposition $o$ is given.

The frame of discernment is defined as ["accurate identification $A$ ", "inaccurate identification $\bar{A}$ " $\}$ in the paper. When all the BPA cannot be assigned to $m(A)$ and $m(\bar{A})$, the rest of BPA is assigned to $\Theta$, that is $m(\Theta)=m(A \cup \bar{A})$. The probability of accurate identification $p_{A}$ is defined as the probability of the identification error less than $15 \%$, which is acquired by analyzing the errors of stress distribution in the key region obtained by the measurements of sensors in the key region and the normal regions. Then, the BPA of $\Theta$ is calculated by the statistical evidence method listed as follows.

Assuming the consensus assumption is met, that is, $\Theta^{0}=\{A, \bar{A}\}$ is an ordered set of $\Theta$, which satisfies $p_{A}>p_{\bar{A}}$, then the corresponding BPA is

$$
\begin{aligned}
& m(A)=\frac{p_{A}-p_{\bar{A}}}{p_{A}} \\
& m(\bar{A})=0 \\
& m(\Theta)=\frac{p_{\bar{A}}}{p_{A}}
\end{aligned}
$$

If the consensus assumption can not be met, the generalization method of acquiring BPA can be achieved by weakening the consensus assumption. 
That is, if $\Theta^{0}=\{A, \bar{A}\}$ is an ordered set of $\Theta$, which satisfies $p_{A}<p_{\bar{A}}$, then the corresponding BPA is

$$
\begin{aligned}
& m(A)=p_{A} \\
& m(\bar{A})=p_{\bar{A}} \\
& m(\Theta)=0
\end{aligned}
$$

After calculating the BPA, the Dempster's rule is used to combine the information. The BPA of fusing the identification results obtained by the measurements of sensors in $i^{\text {th }}$ and $j^{\text {th }}$ regions are given by

$$
\begin{aligned}
& m_{i j}(A)=K\left(m_{i}(A) m_{j}(A)+m_{i}(\Theta) m_{j}(A)+m_{i}(A) m_{j}(\Theta)\right) \\
& m_{i j}(\bar{A})=K\left(m_{i}(\bar{A}) m_{j}(\bar{A})+m_{i}(\Theta) m_{j}(\bar{A})+m_{i}(\bar{A}) m_{j}(\Theta)\right) \\
& m_{i j}(\Theta)=K m_{i}(\Theta) m_{j}(\Theta)
\end{aligned}
$$

Where, $m_{i}$ and $m_{j}$ are BPA of the identification results of the $i^{\text {th }}$ and $j^{\text {th }}$ regions.

$$
K^{-1}=1-\left(m_{i}(A) m_{j}(\bar{A})+m_{i}(\bar{A}) m_{j}(A)\right)
$$

When there are three or more stress distributions obtained by the measurements of sensors in the key regions and the normal regions, the application of Dempster's rule is repeated using the BPA calculated from the first application of the rule and the other BPA from another stress distribution. The Dempster's rule is used to fuse all the possible combination of the stress distributions obtained by measurements of the key regions and the other regions. Finally the combination which has the highest BPA for "accurate identification $A$ " will be selected to calculate the synthesized stress distribution of the key region.

\subsection{Weighted Fusion Algorithm}

The weighted fusion algorithm is used to obtain different fusion coefficients for the key region identification and the normal region identification. If the identified stress of one element in the key region obtained by the measurements of sensors in $\mathrm{n}$ regions are $\sigma_{1}, \sigma_{2}, \cdots \sigma_{n}$, which are independent of each other, and the fusion coefficients corresponding to them are $w_{1}, w_{2}, \cdots, w_{n}$, then the synthesized results is defined as

$$
\hat{\sigma}=\sum_{i=1}^{n} w_{i} \sigma_{i}
$$

where, the fusion coefficients satisfy $\sum_{i=1}^{n} w_{i}=1$.

The fusion coefficients $w$ is determined on the purpose of minimizing the identification errors, which is calculated by minimizing the fitness function with genetic algorithm.

The fitness function is as follows:

$$
f=\sqrt{\frac{\sum_{k=1}^{m}\left(e_{k i}\right)^{2}}{m}}=\sqrt{\frac{\sum_{k=1}^{m}\left[\left(\tilde{\sigma}_{i}-\hat{\sigma}_{i}\right) / \tilde{\sigma}_{i}\right]^{2}}{m}}
$$

where, $m$ is the number of total identified stresses of the $i^{\text {th }}$ elements in the key region with the training samples; $\tilde{\sigma}_{i}$ is the true value of $i^{\text {th }}$ element stress; $\hat{\sigma}_{i}$ is the synthesized stress of $i^{\text {th }}$ element acquired using information fusion, which is given by Eq. (12).

\section{Numerical Simulations}

\subsection{The Finite Element Model}

The model is a Schwedler-type single-layer sphere lattice shell as shown in Fig. 2.

The section of the elements in radial direction is $133 \mathrm{~mm}$ in diameter and $4 \mathrm{~mm}$ in thickness and the section of the elements in ring direction and in oblique direction is $127 \mathrm{~mm}$ in diameter and $3 \mathrm{~mm}$ in thickness. The finite element model of the lattice shell consists

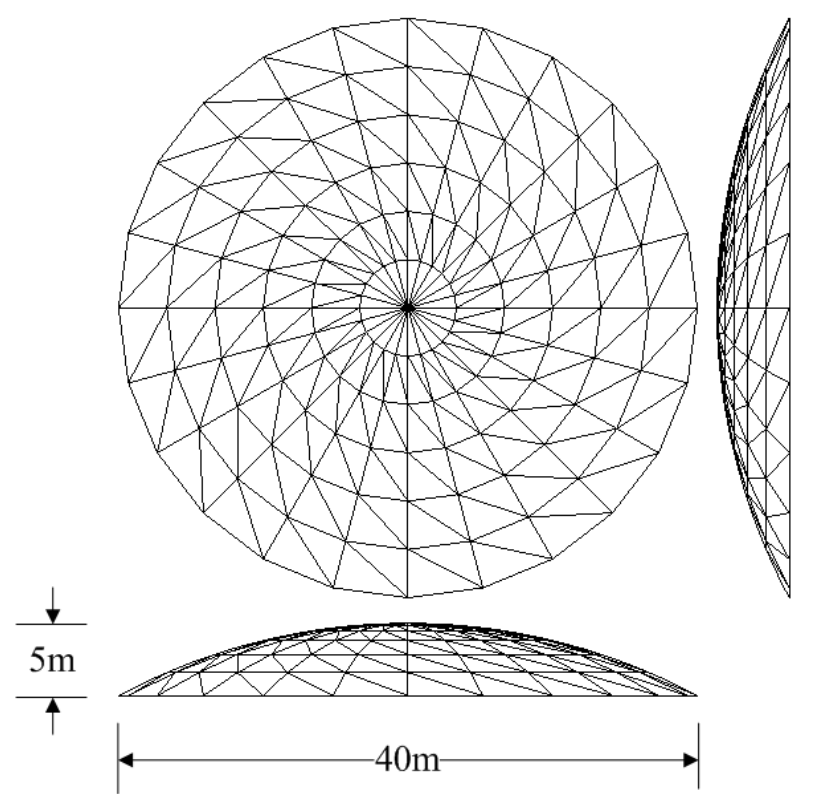

Fig. 2 The finite element model of the lattice shell. 
of 145 nodes, 408 elements, and the supports are fixed supports. In the analysis, the distributed mass is 200 $\mathrm{kg} / \mathrm{m}^{2}$; Young's Modulus is $E=2.06 \times 10^{11} \mathrm{~N} / \mathrm{m}^{2}$; the Poisson ratio is $\mu=0.3$.

\subsection{The Division of the Lattice Shell}

The structure is divided into four regions according to the similarity and the region 1 with 30 elements in ring direction is selected to be the key region for stress identification, which is shown in Fig. 3.

\subsection{The Selection of the Measured Elements}

The measured elements are selected based on the larger means and variation coefficients in each ring of the structure are selected the measured elements, which are shown in Fig. 4 with light color. In addition, the dark colors in Fig. 4 are the unmeasured elements in the key region. Moreover, the measured elements in normal regions have the same arrangements as the key region.

\subsection{The Establishment of Samples}

In order to determine stress distribution patterns, wind loads are simulated with mean wind speeds from $5 \mathrm{~m} / \mathrm{s}$ to $60 \mathrm{~m} / \mathrm{s}$, in every $5 \mathrm{~m} / \mathrm{s}$, a total of 12 different grades in wind loads. The simulation time of wind loads is 10 minutes and the sampling frequency is 10 $\mathrm{Hz}$, so there are 6000 load steps in each grade of wind loads. There are 6000 patterns of stress distributions corresponding to each grade of wind loads from the finite element analysis.

The standard pattern library is formed of 2000 patterns of stress distributions under each grade of wind loads. The training set is formed of 50 patterns of stress distributions under each grade of wind load are extracted to form the training set. The patterns in standard pattern library and the training set are different. The testing set is formed of 50 patterns of stress distributions under the wind load with mean wind speed $42.5 \mathrm{~m} / \mathrm{s}$.

The relative error is used to evaluate the accuracy of the identification. Meanwhile, the elements with

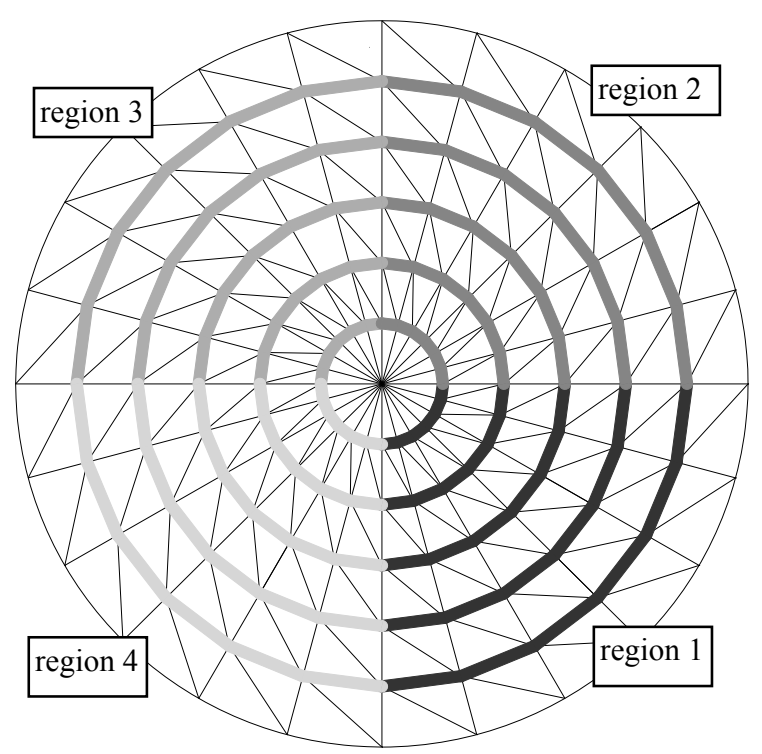

Fig. 3 The division of lattice shell.

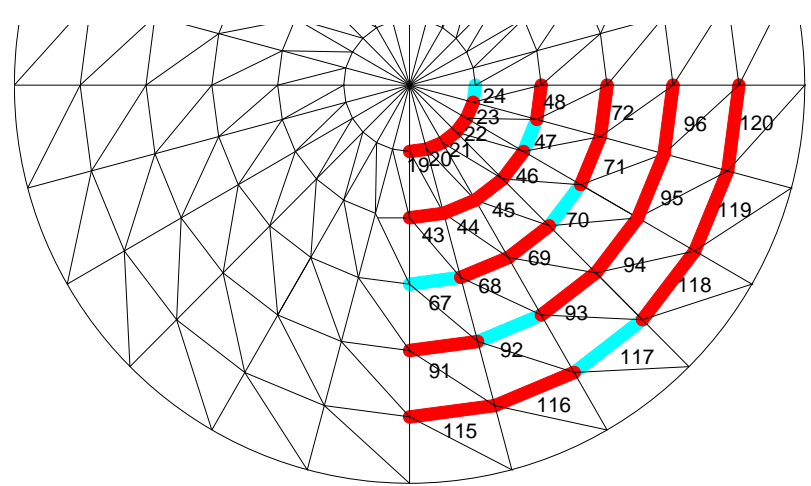

Fig. 4 The arrangement of key regions and measured elements.

large stress value are more important, so the effective of the method is assessed by the identification relative error of these elements. The identification results, in which the stress of the element is more than $100 \mathrm{Mpa}$, are considered to be used as assessment in the effective of the method, while there are 286 identification results.

\subsection{The Key Region Identification}

The identification results of the original method and the improved method using fuzzy neartude is compared and shown in Fig. 5. It can be seen that the number of cases with errors less than $5 \%$ using the improved method is larger than that using the original method and the improved method is much more accurate than the original method. 
When the measurements are disturbed by noise level of $5 \%, 10 \%, 15 \%$ and $20 \%$, which are defined as the ratio of the root mean square (RMS) of the noise to the RMS of stress time series, the identification results of improved method are shown in Fig. 6, the accuracy of identification results is not be affected obviously in the situation of low noise level. However, the accuracy under the disturbing of high noise level is decreased.

\subsection{The Synthesized Stress Distribution of the Key} Region

The corresponding regions for calculating the synthesized stress distribution of the key region is

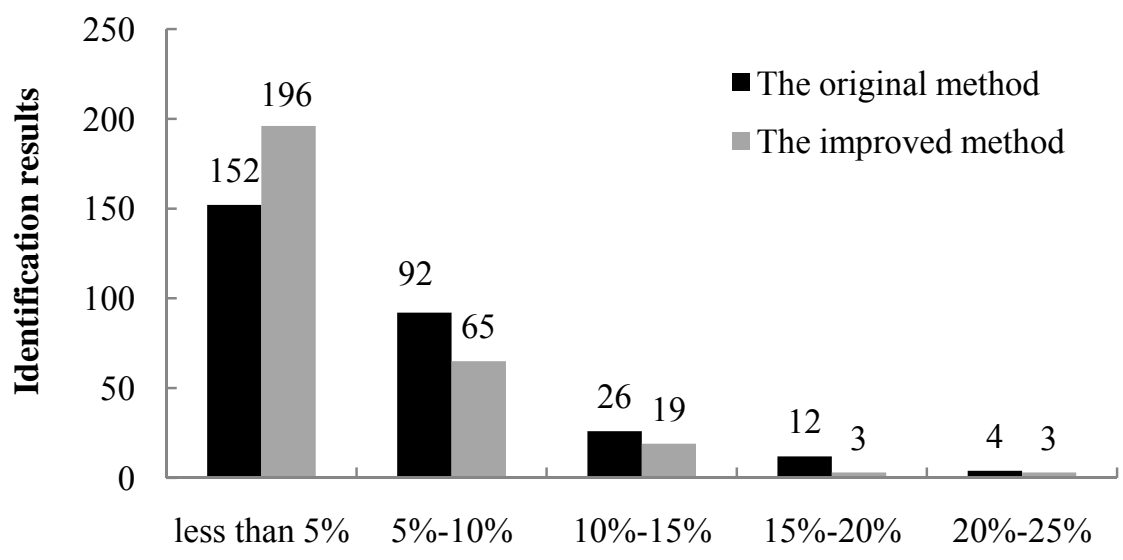

The distribution of errors
The improved method selected by D-S evidence theory and are shown in Table 1. The region in the Table 1 is the corresponding regions selected to calculate the synthesized stress value of the element in the key region.

The identification results without using information fusion is denoted as the identification results before information fusion; the identification results using information fusion is denoted as the identification results after information fusion, which are analyzed and shown in Fig. 7. The results show that the method using information fusion increases the accuracy of identification, but the effectiveness is not very obvious.

Fig. 5 The distribution of errors with fuzzy pattern recognition.

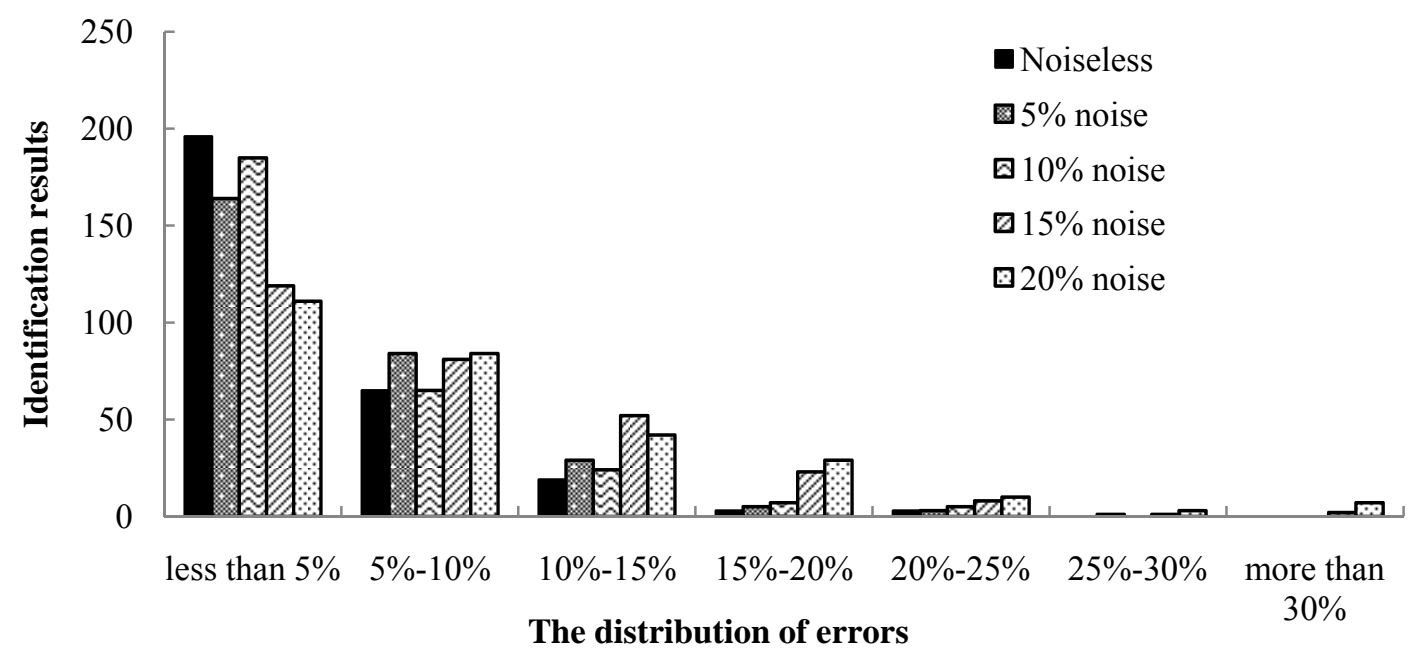

Fig. 6 The distribution of errors with noise. 
When the measurements of sensors are disturbed by noise level of $5 \%, 10 \%, 15 \%$ and $20 \%$, the identification results using information fusion technique are shown in Fig. 8. Comparing with the method without using the information fusion, the identification results are more accuracy under noise disturbing especially in the case of high noise level, as shown in Table 2. The results illustrate that the proposed method using information fusion technique is effective.

Table 1 The regions selected by D-S evidence theory.

\begin{tabular}{|c|c|c|c|c|c|c|c|c|c|}
\hline element & region & element & region & element & region & element & region & element & region \\
\hline 19 & $1 、 4$ & 43 & $1,2 、 4$ & 67 & 1 & 91 & $1 、 2 、 4$ & 115 & $1 、 2 、 4$ \\
\hline 20 & $1 、 2 、 4$ & 44 & $1 、 2 、 3$ & 468 & 1 & 92 & $1 、 2 、 4$ & 116 & $1 、 2 、 4$ \\
\hline 21 & 1 & 45 & $1,2 、 4$ & 69 & $1 、 2 、 4$ & 93 & $1,2 、 4$ & 117 & 1 \\
\hline 22 & 1 & 46 & $1 、 2 、 4$ & 70 & 1 & 94 & 1 & 118 & $1 、 2$ \\
\hline 23 & 1 & 47 & 1 & 71 & $1 、 2 、 4$ & 95 & $1 、 2 、 4$ & 119 & $1 、 2 、 4$ \\
\hline 24 & 1 & 48 & $1,2 、 4$ & 72 & $1 、 2 、 4$ & 96 & $1 、 2 、 4$ & 120 & $1 、 2 、 4$ \\
\hline
\end{tabular}

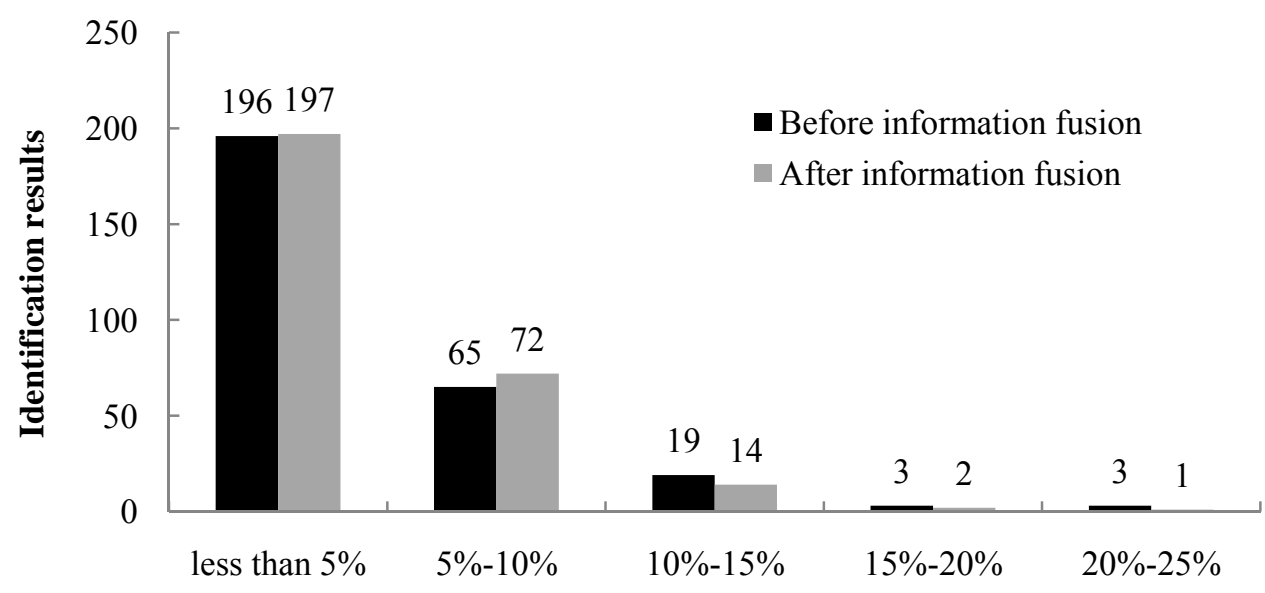

The distribution of errors

Fig. 7 The distribution of errors with information fusion.

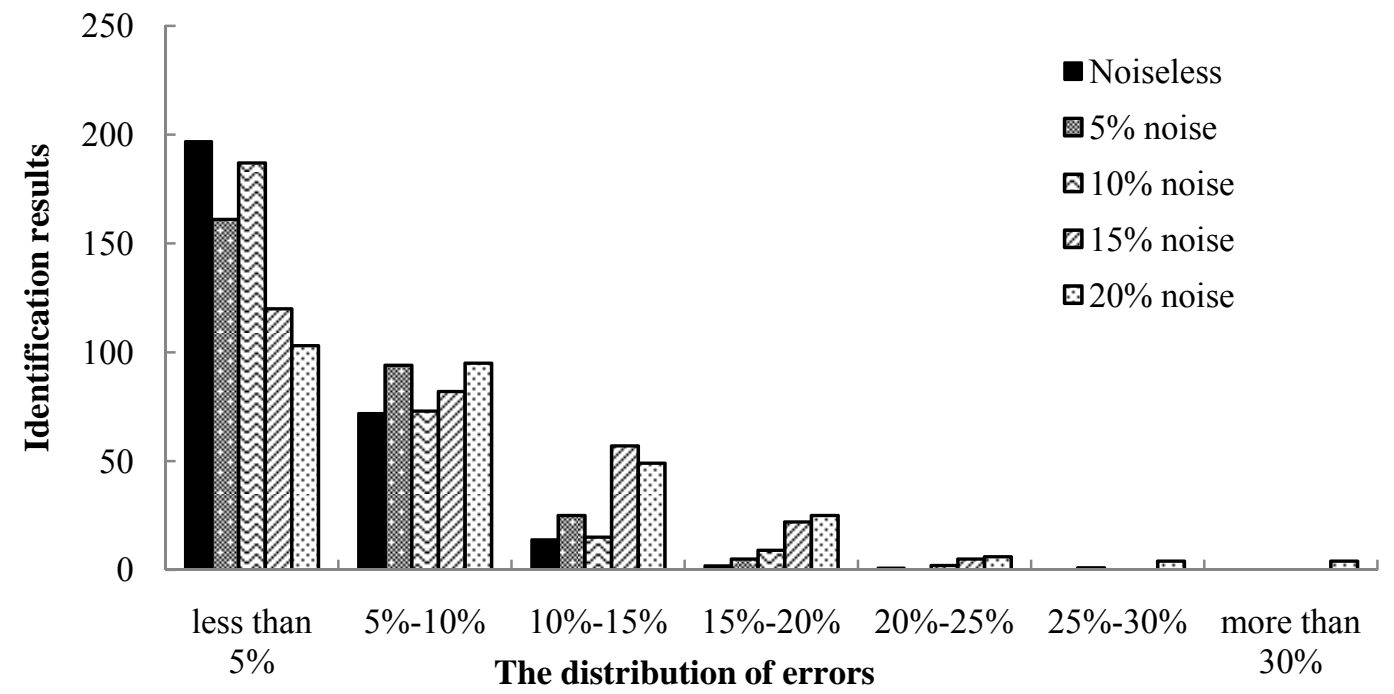

Fig. 8 The distribution of errors with noise. 
Table 2 The comparison of identification results with noise.

\begin{tabular}{ccccc}
\hline \multirow{2}{*}{ Noise level } & \multicolumn{2}{c}{ Before information fusion } & \multicolumn{2}{c}{ After information fusion } \\
\cline { 2 - 4 } & $\begin{array}{c}\text { The ratio of errors less } \\
\text { than } 15 \%\end{array}$ & $\begin{array}{l}\text { The maximum error } \\
\text { than } 15 \%\end{array}$ & $98.95 \%$ & $23.20 \%$ \\
\hline 0 & $97.90 \%$ & $23.20 \%$ & $97.90 \%$ & $25.15 \%$ \\
$5 \%$ noise & $96.85 \%$ & $25.16 \%$ & $96.15 \%$ & $23.27 \%$ \\
$10 \%$ noise & $95.80 \%$ & $23.20 \%$ & $90.56 \%$ & $23.88 \%$ \\
$15 \%$ noise & $88.11 \%$ & $33.60 \%$ & $86.36 \%$ & $32.99 \%$ \\
$20 \%$ noise & $82.87 \%$ & $36.25 \%$ & The maximum error \\
\hline
\end{tabular}

\section{Conclusions}

The stress identification method using the measurements of sensors in the whole structure is proposed. An improved fuzzy pattern recognition method introducing the fuzzy neartudes as the weighting coefficients is discussed. The measurements of sensors in the normal regions are used to improve the accuracy of stress identification in the key region by information fusion technique. The following conclusions can be obtained:

(1) The original fuzzy pattern recognition can be used to identify stresses of elements with limited measurements of sensors, but the identification accuracy is not very good.

(2) The usage of improved fuzzy pattern recognition and information fusion technology can improve the reliability of stress identification.

(3) The D-S evidence theory is effective in selecting the normal region identification, and the weighted fusion algorithm can be used to acquire the fusion coefficients of each region. But these two methods could only be used in the early-stage preparation part with data from the finite element model.

(4) When the measurements of sensors are disturbed by noise, especially in the case of high noise level, the use of information fusion technique will bring in more reliable results.

\section{Acknowledgements}

The work presented in this paper was partially supported by Natural Science Foundation of China (NSFC No. 50678052) and Key Projects in the
National Science \& Technology Pillar Program during the Eleventh Five-year Plan Period of China (No. 2006BAJ13B03).

\section{References}

[1] S. C. Liu and D. S. Jiang, The application of structural health monitoring system technology FBG-based to No.2 Wuhan Bridge over Yangtze River, Photonics and Optoelectronics Meetings, Fiber Optic Communication and Sensors, Proceedings of SPIE 72780O, 2008.

[2] F. N. Catbas, M. Susoy and D. M. Frangopol, Structural health monitoring and reliability estimation: Long span truss bridge application with environmental monitoring data, Engineering Structures 30 (2008) 2347-2359.

[3] T. H. T. Chan, L. Yu, H. Y. Tam, Y. Q. Ni, S. Y. Liu, W. H. Chung and L. K. Cheng, Fiber Bragg Grating sensors for structural health monitoring of Tsing Ma Bridge: background and experimental observation, Engineering Structures 28 (2006) 648-659.

[4] C. R. Farrar, H. Sohn, M. L. Fugate and J. J. Czarnecki, Integrated structural health monitoring, Advanced Nondestructive Evaluation for Structural and Biological Health Monitoring, Proceedings of SPIE, Vol. 4335, 2001.

[5] P. J. Barr, C. B. Woodward, B. Najera and M. N. Amin, Long-term structural gealth monitoring of the San Ysidro Bridge, Journal of Performance of Constructed Facilities 20 (1) (2006) 14-20.

[6] M. Liu, D. M. Frangopol and S. Y. Kim, Bridge system performance assessment from structural health monitoring: A case study, Journal of Structural Engineering 35 (6) (2009) 733-742.

[7] J. Wang, W. L. Qu, J. Zhen and H. Liu, Fuzzy pattern identification and experimental verification of stress field of space steel structure, International Conference on Health Monitoring of Structure, Material and Environment, 2007, pp. 687-793

[8] H. Y. Guo, Structural damage detection using information fusion technique, Mechanical Systems and Signal Processing 20 (5) (2006) 1173-1188. 
[9] H. Li, Y. Q. Bao and J. P. Ou, Structural damage identification based on integration of information fusion and shannon entropy, Mechanical System and Signal Processing 22 (6) (2008) 1427-1440

[10] Y. Xu, K. Y. Qin, J. Liu and Z. M. Song, Fuzzy pattern recognition and its application, Xi'an Jiaotong University, 1998.
[11] L. A. Klein, Sensor and data fusion, The International Society for Optical Engineering, 2004.

[12] Y. He, G. H. Wang, D. J. Lu and Y. N. Peng, Multisensor information fusion with applications, Publishing House of Electronics Industry, 2009. 